\title{
Bile acid promotes liver regeneration via farnesoid $X$ receptor signaling pathways in rats
}

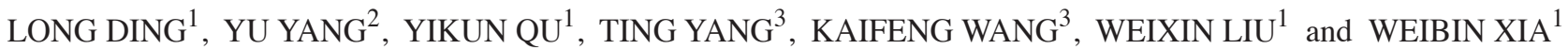 \\ ${ }^{1}$ The Second Department of General Surgery, The First Affiliated Hospital of Jiamusi University; \\ ${ }^{2}$ Department of Topographical Anatomy, College of Basic Medical Sciences, Jiamusi University; \\ ${ }^{3}$ Department of Vascular Surgery, The First Affiliated Hospital of Jiamusi University, Jiamusi, Heilongjiang 154003, P.R. China
}

Received February 17, 2014; Accepted October 29, 2014

DOI: $10.3892 / \mathrm{mmr} .2015 .3270$

\begin{abstract}
Bile acids, which are synthesized from cholesterol in the hepatocytes of the liver, are amphipathic molecules with a steroid backbone. Studies have shown that bile acid exhibits important effects on liver regeneration. However, the mechanism underlying these effects remains unclear. The aim of the present study was to investigate the effect of bile acid and the farnesoid X receptor (FXR) on hepatic regeneration and lipid metabolism. Rats were fed with $0.2 \%$ bile acid or glucose for 7 days and then subjected to a 50 or $70 \%$ hepatectomy. Hepatic regeneration rate, serum and liver levels of bile acid, and expression of FXR and Caveolin-1, were detected at 24, 48 or $72 \mathrm{~h}$ following hepatectomy. The expression of proliferating cell nuclear antigen (PCNA) in the liver was measured using immunohistochemistry at the end of the study. Hepatocytes isolated from rats were treated with bile acid, glucose, FXR agonist and FXR antagonist, separately or in combination. Lipid metabolism, the expression of members of the FXR signaling pathway and energy metabolism-related factors were measured using ELISA kits or western blotting. Bile acid significantly increased the hepatic regeneration rate and the expression of FXR, Caveolin-1 and PCNA. Levels of total cholesterol and high density lipoprotein were increased in bile acid- or FXR agonist-treated hepatocytes in vitro. Levels of triglyceride, low density lipoprotein and free fatty acid were decreased. In addition, bile acid and FXR agonists increased the expression of bile salt export pump and small heterodimer partner, and downregulated the expression of apical sodium-dependent bile acid transporter, $\mathrm{Na}^{+} /$taurocholate cotransporting polypeptide and cholesterol $7 \alpha$-hydroxylase. These results suggested that physiological concentrations of bile acid may promote liver regeneration via FXR signaling pathways, and may be associated with energy metabolism.
\end{abstract}

Correspondence to: Dr Long Ding, The Second Department of General Surgery, The First Affiliated Hospital of Jiamusi University, 234 Dexiang Street, Jiamusi, Heilongjiang 154003, P.R. China

E-mail: jmsdinglong@163.com

Key words: bile acid, farnesoid $\mathrm{X}$ receptor, liver regeneration

\section{Introduction}

Liver regeneration is important in the recovery from injury induced by surgery, trauma, poisoning, infection, necrosis or liver transplantation (1). Consequently, research investigating the improvement of the regeneration ability of the liver, is of great significance. During regeneration, quiescent mature hepatocytes reenter the cell cycle in order to proliferate and divide, thus leading to hepatic regeneration without the involvement of stem cells. Although the exact mechanisms have not been fully characterized, a study demonstrated that liver regeneration primarily comprises cell proliferation, lipid metabolism, various growth factors, and a number of cytokines and their signaling pathways (2).

Bile acid, which is synthesized from cholesterol, is the chief components of bile. The primary functions of bile are to digest the fat soluble molecules in food and to aid in the intestinal absorption of lipids in vivo. Recent studies have shown that bile acid acts as a signaling molecule by activating signaling pathways, and that it participates in the process of liver regeneration $(3,4)$. A number of transport proteins for bile acid have been identified in the liver and are known to be regulated by nuclear receptors (5). Nuclear receptors (NRs) are ligand-activated transcription factors that are members of a super family, consisting of 48 proteins (6). The farnesoid X receptor (FXR), a member of the sub-cluster of metabolic NRs, was originally isolated from a rat liver cDNA library and cloned in 1995 (7). FXR is predominantly expressed in the liver, kidney, intestine and adrenal glands, and is involved in regulating the metabolism of bile acid and cholesterol $(8,9)$. Furthermore, the interaction of bile acid with FXR is essential for glucose metabolism, liver inflammation and liver regeneration (10-12).

In the current study, bile acid, glucose and FXR were administrated in vivo and in vitro, and the effects of these molecules on liver regeneration and lipid metabolism were compared. The mechanisms underlying these effects were also explored.

\section{Materials and methods}

Animals. Male Sprague-Dawley rats (weight, 250-300 g) were obtained from the Experimental Animal Center of Jiamusi 
University (Jiamusi, China) and housed in a temperature- and light-controlled room at $19-22^{\circ} \mathrm{C}$ with a $12 \mathrm{~h}$ light/dark cycle. The animals had free access to food and water. The animal study protocol was reviewed and approved by the Institutional Animal Care and Use Committee of Jiamusi University.

Rat model of hepatectomy. Rats were fasted for $12 \mathrm{~h}$ and assigned to one of the following three groups: Control group (rats were fed with normal diet), bile acid group (rats were fed with normal diet plus $0.2 \%$ bile acid) and glucose group (rats were fed with normal diet plus $10 \%$ glucose). There were 36 rats in each group. At day 7, rats were subjected to 50 or $70 \%$ hepatectomy and the weight of removed liver tissues was recorded as $\mathrm{mR}$. At 24, 48 or $72 \mathrm{~h}$ following hepatectomy, the rats were sacrificed by cervical dislocation following anesthesia induction, and liver tissues and blood were collected immediately. Total liver mass was calculated based on the ratio of removed liver weight and volume, and recorded as $\mathrm{mT}$. That is, for rats in which $50 \%$ of the was liver removed, the weight of the removed portion was divided by 0.5 to obtain the total volume, and for those in which $70 \%$ was removed, it was divided by 0.7 . The liver weight of sacrificed rats was recorded as $\mathrm{mS}$ and the hepatic regeneration rate was calculated as $[\mathrm{mS}-(\mathrm{mT}-\mathrm{mR})] / \mathrm{mT} \times 100$.

Immunohistochemistry. Fresh liver samples were immediately fixed in neutral formalin and embedded in paraffin. Paraffin-embedded liver samples were cut into 5- $\mu \mathrm{m}$ sections deparaffinized in xylene, rehydrated in serial dilutions of ethanol, placed in antigen retrieval solution and microwaved at low power for $10 \mathrm{~min}$. The activity of endogenous peroxidase was blocked by incubation with $3 \%$ $\mathrm{H}_{2} \mathrm{O}_{2}$ (Sinopharm Chemical Reagent, Shanghai, China). A mouse monoclonal antibody against proliferating cell nuclear antigen (PCNA; 1:50; sc-252820; Santa Cruz Biotechnology, Inc., Santa Cruz, CA, USA) was added and incubated at $4^{\circ} \mathrm{C}$ overnight. Sections were then incubated with a horseradish peroxidase (HRP)-conjugated goat anti-mouse antibody (1:200; A0216; Beyotime Institute of Biotechnology, Haimen, China) for $30 \mathrm{~min}$ at $37^{\circ} \mathrm{C}$. Positive signals were visualized by 3,3'-Diaminobenzidine (Solarbio, Beijing, China) and sections were then counterstained with hematoxylin. Images of the sections were captured using a microscope (x400; BX51, Olympus, Tokyo, Japan). A brown or yellow color was regarded as a positive reaction.

Isolation of hepatocytes and experimental setup. Hepatocyte isolation was performed as previously described (13). Briefly, following administration of anesthesia with $10 \%$ chloral hydrate (Sinopharm Chemical Reagent), a cannula was introduced into the portal vein and D-Hank's solution (Solarbio) was perfused in order to remove the blood. Collagenase (0.05\%; Invitrogen, Carlsbad, CA, USA) was then perfused to hydrolyse the collagen molecules. The liver was removed from the capsule, cut into sections and passed through a 200 mesh sieve. The viability of isolated hepatocytes was $>95 \%$, as assessed by trypan blue (Beyotime Institute of Biotechnology) exclusion. Primary hepatocytes were cultured in Dulbecco's modified Eagle's medium (DMEM, Gibco, Grand Island, NY, USA) supplemented with $10 \%$ fetal bovine serum (FBS;
Hyclone, Logan, UT, USA) at $37^{\circ} \mathrm{C}$ in a humidified $5 \% \mathrm{CO}_{2}$ atmosphere. The medium was replaced every 2 days, and cells were passaged when they reached $80-90 \%$ confluence. The experiments were initiated following the third passage, with cells being divided into nine groups: The control group (hepatocytes were cultured in normal medium); the bile acid group (hepatocytes were cultured in normal medium with $40 \mu \mathrm{mol} / 1$ bile acid; Sigma-Aldrich, St. Louis, MO, USA); the glucose group (hepatocytes were cultured in normal medium with $25 \mathrm{mmol} / 1$ glucose); the FXR agonist group (hepatocytes were cultured in normal medium with $10 \mu \mathrm{mol} / \mathrm{l} \mathrm{FXR}$ agonist, GW4064; Sigma-Aldrich); the FXR antagonist group (hepatocytes were cultured in normal medium with $100 \mu \mathrm{mol} / 1$ FXR antagonist, Guggulsterones; Sigma-Aldrich); the bile acid and FXR agonist group (hepatocytes were cultured in normal medium with $40 \mu \mathrm{mol} / 1$ bile acid and $10 \mu \mathrm{mol} / 1 \mathrm{FXR}$ agonist, GW4064); the bile acid and FXR antagonist group (hepatocytes were cultured in normal medium with $40 \mu \mathrm{mol} / 1$ bile acid and $100 \mu \mathrm{mol} / \mathrm{l} \mathrm{FXR}$ antagonist, Guggulsterones); the glucose and FXR agonist group (hepatocytes were cultured in normal medium with $25 \mathrm{mmol} / \mathrm{l}$ glucose and $10 \mu \mathrm{mol} / \mathrm{l} \mathrm{FXR}$ agonist, GW4064); and the glucose and FXR antagonist group (hepatocytes were cultured in normal medium with $25 \mathrm{mmol} / 1$ glucose and $100 \mu \mathrm{mol} / 1 \mathrm{FXR}$ antagonist, Guggulsterones). Cells were incubated at $37^{\circ} \mathrm{C}$ with $5 \% \quad \mathrm{CO}_{2}$ and saturated humidity conditions in a culture box (Heal Force, Shanghai. China) and collected at $72 \mathrm{~h}$.

Analysis of total bile acid (TBA), triglyceride (TG), total cholesterol (TC), high density lipoprotein (HDL), low density lipoprotein (LDL) and free fatty acid (FFA). The levels of TBA in liver and serum were measured with the TBA assay kit (Nanjing Jiancheng Bioengineering Institute, China) according to manufacturer's instructions. The levels of TG, TC, HDL, LDL and FFA in hepatocytes were assessed using an ELISA kit (Rat TG ELISA kit, Ximei, Shanghai, China; Rat TC ELISA kit, Rat HDL ELISA kit, Hyperheal, Shanghai, China; Rat LDL ELISA kit, J\&L Biological, Shanghai, China; Rat FFA ELISA kit, Lianshuo Biological, Shanghai, China, respectively). Optical density was measured using a microplate reader (BioTek Instruments, Winooski, VT, USA).

Western blotting. Total liver protein was extracted with a radioimmunoprecipitation assay lysis buffer (Beyotime Institute of Biotechnology, China) and total cellular protein was extracted with NP-40 buffer (Beyotime Institute of Biotechnology). Equal quantities of total protein $(40 \mu \mathrm{g})$ were loaded and separated using $10 \%$ sodium dodecyl sulfate-polyacrylamide gel electrophoresis, transferred to polyvinylidene fluoride membranes (EMD Millipore, Billerica, MA, USA) and blocked in 5\% fat-free milk for $1 \mathrm{~h}$. Membranes were incubated with primary antibody at $4{ }^{\circ} \mathrm{C}$ overnight. The following primary antibodies were used: A rabbit polyclonal antibody to FXR (1:100; sc-13063; Santa Cruz Biotechnology, Inc.), a rabbit polyclonal antibody to Caveolin 1 [1:1,000 (animal) or 1:2,000 (cell); ab2910; Abcam, Cambridge, UK], a rabbit polyclonal antibody to ASBT (1:1,000; bs-4189R; Bioss, Beijing, China), a rabbit polyclonal antibody to $\operatorname{BSEP}(1: 1,000 ;$ ab99088), a rabbit monoclonal antibody to NTCP $(1: 1,000 ;$ ab133670) (Abcam) 


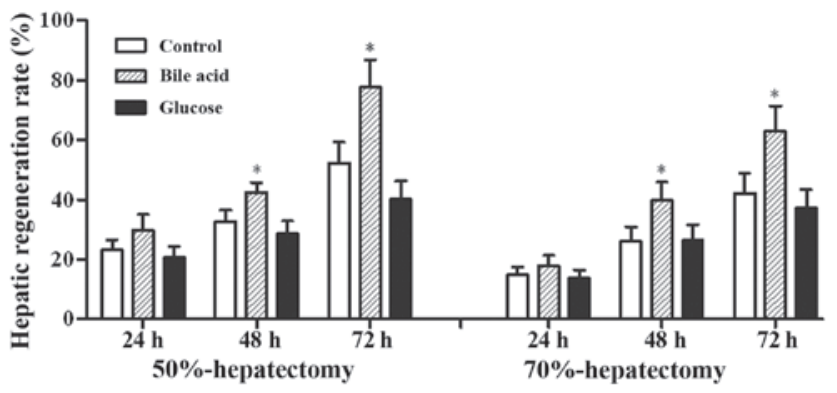

Figure 1. Hepatic regeneration rate of rats following partial hepatectomy. Rats were fed with $0.2 \%$ bile acid or $10 \%$ glucose for 7 days and subjected to $50 \%$ or $70 \%$ hepatecomy. Hepatic regeneration rates were detected at 24,48 or $72 \mathrm{~h}$ after hepatectomy. Data are presented as the mean \pm standard deviation, $\mathrm{n}=6$. ${ }^{*} \mathrm{P}<0.05$ and ${ }^{* *} \mathrm{P}<0.01$, compared with the control group.

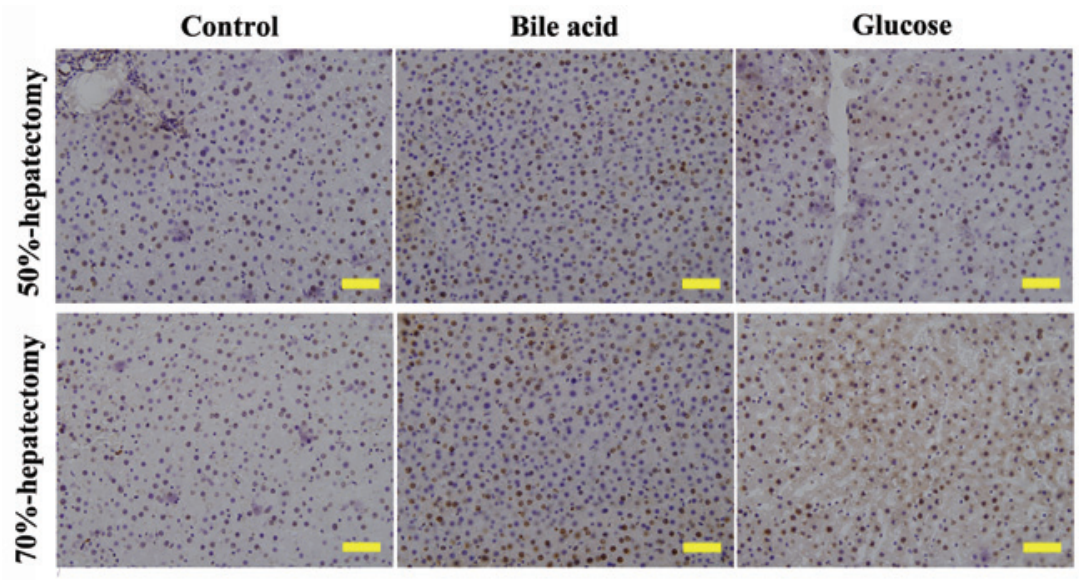

Figure 2. Expression of PCNA in regenerating liver. Rats were fed with $0.2 \%$ bile acid or $10 \%$ glucose for 7 days and subjected to $50 \%$ or $70 \%$ hepatecomy. Expression of PCNA was detected by immunohistochemistry $72 \mathrm{~h}$ after hepatectomy. Positive signals were visualized by 3,3 '-diaminobenzidine and sections were then counterstained with hematoxylin (scale bar, $50 \mu \mathrm{m}$ ). PCNA, proliferating cell nuclear antigen.
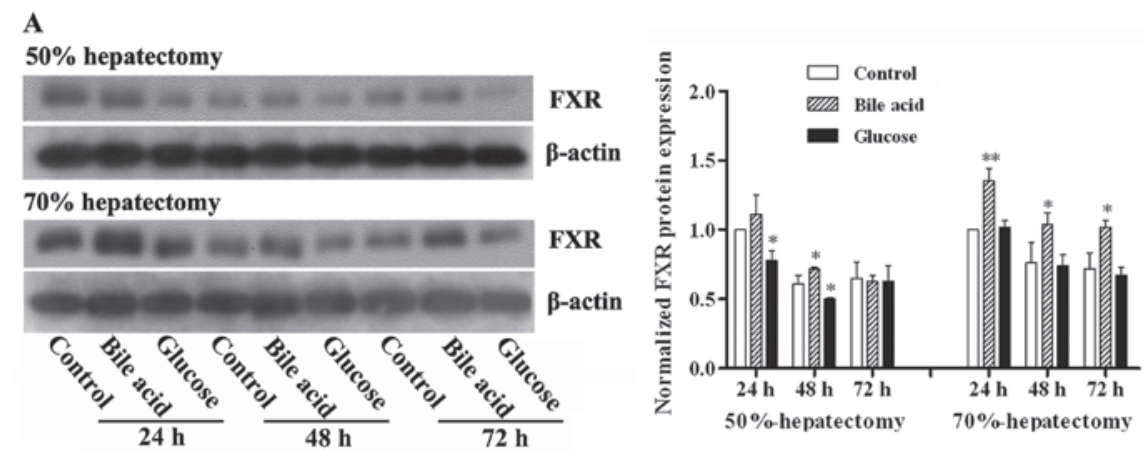

B
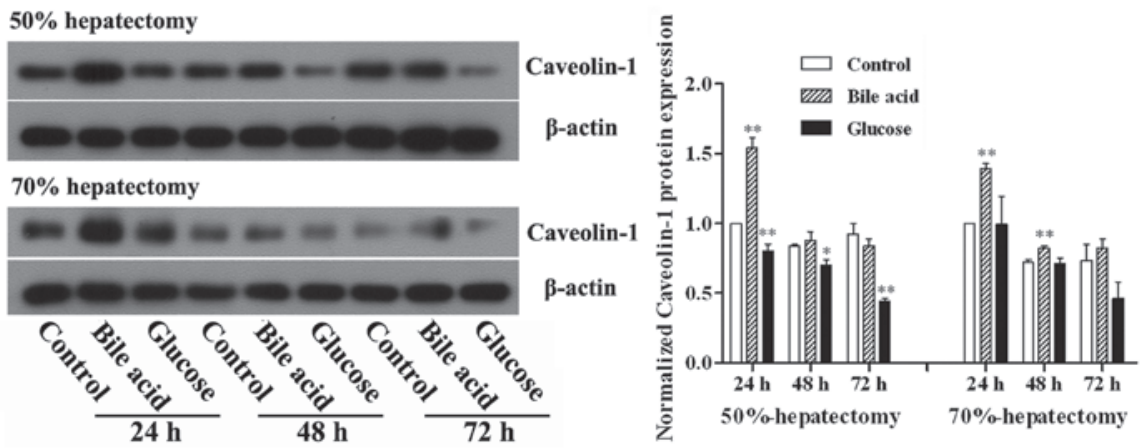

Figure 3. Expression of FXR and Caveolin-1 in liver. Protein level of (A) FXR and (B) Caveolin-1 were detected by western blotting and normalized by gradation analysis. $\beta$-actin was used as an internal control. Data are presented as the mean \pm standard deviation, $\mathrm{n}=6$. ${ }^{*} \mathrm{P}<0.05$ and ${ }^{* *} \mathrm{P}<0.01$, compared with control. FXR, farnesoid $\mathrm{X}$ receptor. 
Table I. Changes of lipid metabolism related factors in hepatocytes in vitro.

\begin{tabular}{lccccc}
\hline Group & TG $(\mu \mathrm{g})$ & TC $(\mathrm{mmol})$ & HDL $(\mu \mathrm{mol})$ & LDL $(\mu \mathrm{mol})$ & FFA $(\mu \mathrm{mol})$ \\
\hline Control & $36.59 \pm 0.70$ & $0.66 \pm 0.03$ & $1.15 \pm 0.02$ & $0.90 \pm 0.01$ & $1.67 \pm 0.03$ \\
Bile acid & $20.85 \pm 0.35^{\mathrm{b}}$ & $0.81 \pm 0.05^{\mathrm{a}}$ & $2.58 \pm 0.07^{\mathrm{b}}$ & $0.70 \pm 0.01^{\mathrm{b}}$ & $1.21 \pm 0.02^{\mathrm{b}}$ \\
Glucose & $42.58 \pm 1.87^{\mathrm{a}}$ & $0.45 \pm 0.05^{\mathrm{b}}$ & $0.96 \pm 0.10^{\mathrm{a}}$ & $0.92 \pm 0.02$ & $1.62 \pm 0.18$ \\
FXR agonist & $17.50 \pm 0.64^{\mathrm{b}}$ & $0.90 \pm 0.00^{\mathrm{b}}$ & $2.77 \pm 0.10^{\mathrm{b}}$ & $0.73 \pm 0.02^{\mathrm{b}}$ & $1.24 \pm 0.05^{\mathrm{b}}$ \\
FXR antagonist & $53.37 \pm 2.32^{\mathrm{b}}$ & $0.34 \pm 0.07^{\mathrm{b}}$ & $0.83 \pm 0.04^{\mathrm{b}}$ & $1.09 \pm 0.00^{\mathrm{b}}$ & $2.03 \pm 0.01^{\mathrm{b}}$ \\
Bile acid+FXR agonist & $16.03 \pm 0.59^{\mathrm{b}}$ & $0.94 \pm 0.03^{\mathrm{b}}$ & $2.93 \pm 0.05^{\mathrm{b}}$ & $0.67 \pm 0.02^{\mathrm{b}}$ & $1.12 \pm 0.04^{\mathrm{b}}$ \\
Bile acid+FXR antagonist & $48.29 \pm 2.51^{\mathrm{b}}$ & $0.43 \pm 0.05^{\mathrm{b}}$ & $0.96 \pm 0.07^{\mathrm{a}}$ & $1.04 \pm 0.03^{\mathrm{b}}$ & $1.92 \pm 0.07^{\mathrm{b}}$ \\
Glucose+FXR agonist & $25.13 \pm 1.13^{\mathrm{b}}$ & $0.69 \pm 0.07$ & $1.75 \pm 0.10^{\mathrm{b}}$ & $0.73 \pm 0.02^{\mathrm{b}}$ & $1.23 \pm 0.04^{\mathrm{b}}$ \\
Glucose+FXR antagonist & $58.45 \pm 1.88^{\mathrm{b}}$ & $0.20 \pm 0.00^{\mathrm{b}}$ & $0.69 \pm 0.03^{\mathrm{b}}$ & $1.12 \pm 0.00^{\mathrm{b}}$ & $2.12 \pm 0.02^{\mathrm{b}}$
\end{tabular}

Hepatocytes were treated with bile acid, glucose, FXR agonist or antagonist for $72 \mathrm{~h}$. Data are presented as the mean \pm standard deviation, $\mathrm{n}=3$. ${ }^{\mathrm{a}} \mathrm{P}<0.05$ and ${ }^{\mathrm{b}} \mathrm{P}<0.01$ compared with control. TG, triglyceride; TC, total cholesterol; HDL, high density lipoprotein; LDL, low density lipoprotein; FAA, free fatty acid; FXR, farnesoid X receptor.

a goat polyclonal antibody to SHP (1:100; sc-15283) and a rabbit polyclonal antibody to CYP7A1 (1:100; sc-25536) (Santa Cruz Biotechnology, Inc.). Membranes were then washed and incubated with goat anti-rabbit (A0208) or donkey anti-goat (A0108) HRP-conjugated secondary antibodies (1:5,000, Beyotime Institute of Biotechnology) at $37^{\circ} \mathrm{C}$ for $45 \mathrm{~min}$. Immunoreactive bands were visualized by enhanced chemiluminescence solution (Qihai Biotech, Shanghai, China) according to the manufacturer's instructions.

Statistical analysis. Data are presented as the mean \pm standard deviation. Two-tailed Student's t test was used to assess statistical significance. Data were analyzed using GraphPad Prism 5.0 software (GraphPad Software, Inc., San Diego, CA, USA). $\mathrm{P}<0.05$ was considered to indicate a statistically significant difference.

\section{Results}

Hepatic regeneration rate and expression of $P C N A$. The restituted liver mass in the bile acid group was markedly increased, with a peak at $72 \mathrm{~h}$, and was higher than that in the control group. By contrast, the hepatic regeneration rate of the rats fed with glucose was reduced compared with the control group (Fig. 1). The calculated regeneration rate of rats that survived $70 \%$ liver resection was less than that in the $50 \%$ resection group (survival, $n=6$ per group). PCNA is a protein marker of DNA synthesis that is involved in the initiation of cell proliferation. The expression of PCNA is correlated with the S-phase of the cell cycle. At $72 \mathrm{~h}$ following hepatectomy, the expression of PCNA was highest in the bile acid group (Fig. 2), which was in accordance with the results of the hepatic regeneration rate.

Expression of FXR and Caveolin-1 in liver. FXR is known to be an important receptor for bile acid and its activation affects lipid and glucose metabolism (14). Caveolin-1 is involved in the regulation of intracellular homeostasis, such as lipid metabolism, cell activation and cell proliferation (15). Based
A

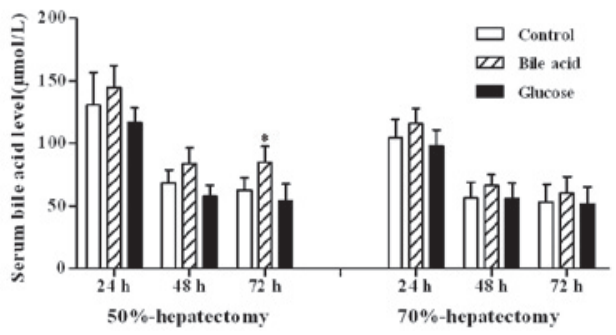

B

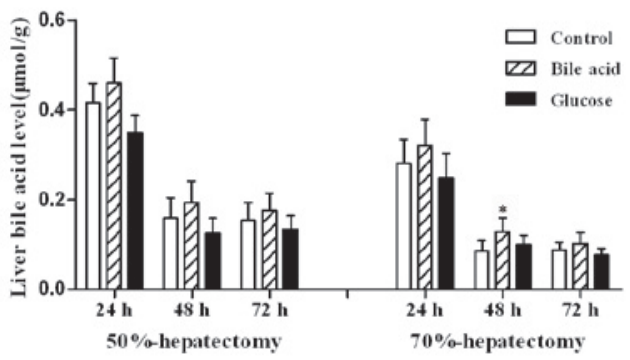

Figure 4. Bile acid levels in serum and liver. Levels of (A) serum bile acid and (B) liver bile acid were measured using the total bile acid assay kit. Data are presented as the mean \pm standard deviation, $\mathrm{n}=6$. ${ }^{*} \mathrm{P}<0.05$ and ${ }^{* *} \mathrm{P}<0.01$, compared with control.

on the results of the initial experiments, which demonstrated the facilitation of hepatic regeneration by bile acid, the effect of bile acid on the expression of FXR and Caveolin-1 was investigated. As shown in Fig. 3, the protein expression of FXR and Caveolin-1 was elevated in response to administration of bile acid. By contrast, treatment with glucose decreased the expression of FXR and Caveolin-1.

Bile acid levels following hepatectomy. Serum and liver bile acid levels were similar to those measured in a previous study (16). Oral administration of $0.2 \%$ bile acid for 7 days significantly increased the concentration of serum and liver bile acid, whereas the concentration decreased in the glucose-fed group (Fig. 4). 
A

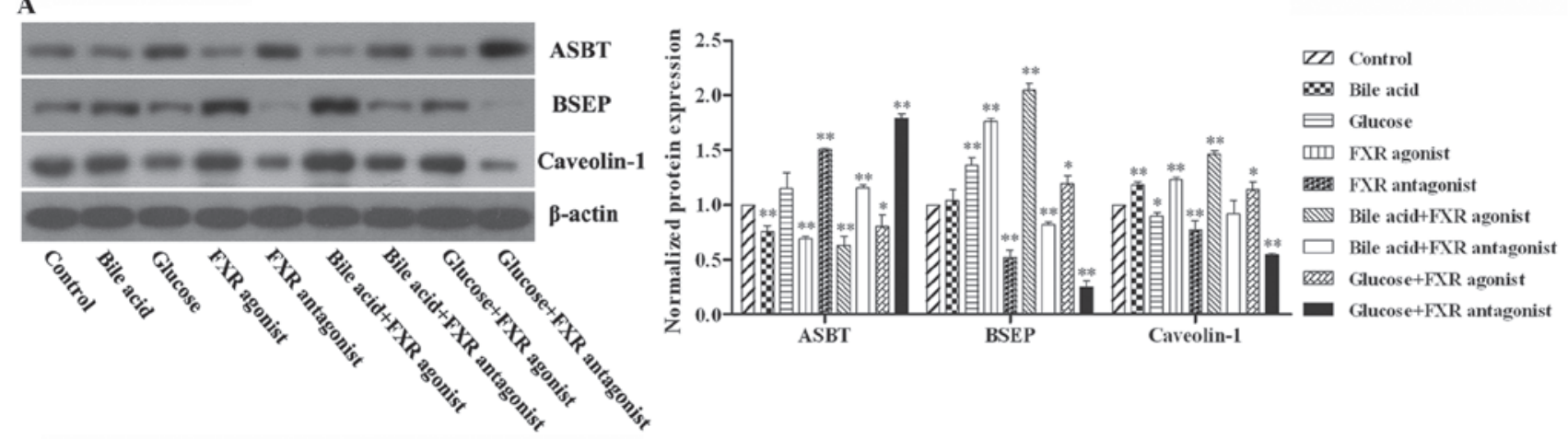

B
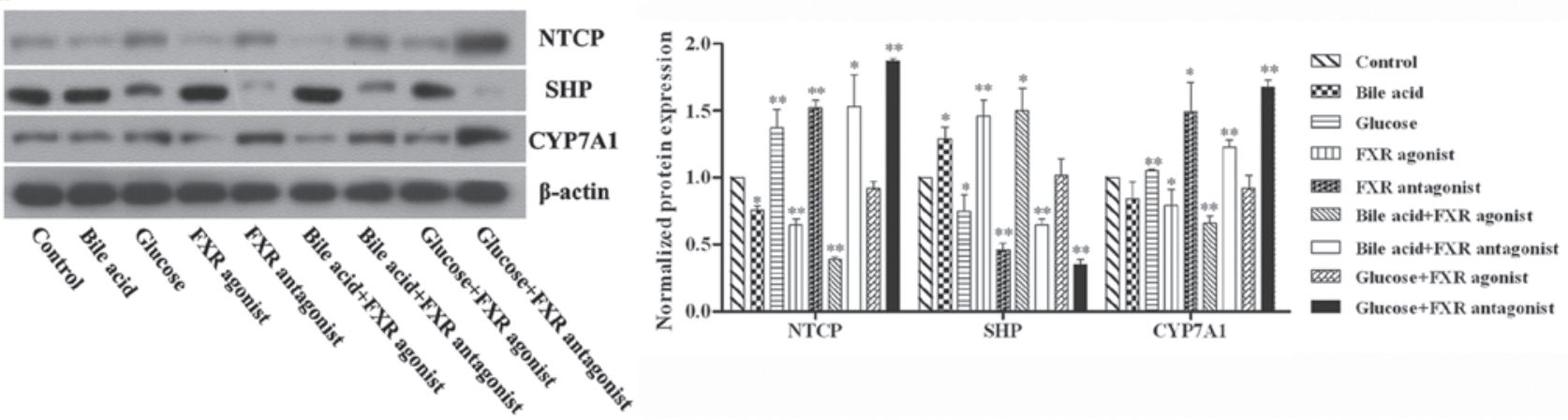

Figure 5. FXR signaling- and lipid metabolism-associated proteins in hepatocytes. Hepatocytes isolated from the rats were treated with bile acid, glucose, FXR agonist or antagonist for $72 \mathrm{~h}$. Expression of (A) ASBT, BSEP, Caveolin-1 protein and (B) NTCP, SHP, CYP7A1 in the hepatocytes were detected using western blotting. Normalized expression was analyzed by gradation. Data are presented as the mean \pm standard deviation, $\mathrm{n}=6$, ${ }^{*} \mathrm{P}<0.05$ and ${ }^{* * *} \mathrm{P}<0.01$ compared with control. FXR, farnesoid X receptor; ASBT, apical sodium-dependent bile acid transporter; BSEP, bile salt export pump; $\mathrm{NTCP}, \mathrm{Na}^{+} /$taurocholate cotransporting polypeptide and cholesterol $7 \alpha$-hydroxylase; SHP, small heterodimer partner.

Changes in levels of lipid metabolism-related factors in hepatocytes in vitro. In order to investigate the effects of bile acid and FXR on lipid metabolism in hepatocytes, TG, TC, HDL, LDL and FFA levels in liver cells were measured (Table I). Levels of TG, LDL and FFA were reduced in the bile acid and FXR agonist groups, whilst they were increased in the glucose and FXR antagonist groups. By contrast, levels of TC and HDL were increased in the bile acid and FXR agonist groups, and decreased in the glucose and FXR antagonist groups. These differences were statistically significant $(\mathrm{P}<0.05)$.

Expression of FXR signaling-related proteins in hepatocytes. In order to verify the mechanism by which bile acid and FXR affect lipid metabolism, the expression of FXR signaling- and lipid metabolism-associated proteins was determined. As shown in Fig. 5, the expression of bile salt export pump (BSEP), Caveolin-1 and small heterodimer partner (SHP) were elevated in response to bile acid and FXR agonist, whereas that of apical sodium-dependent bile acid transporter (ASBT), $\mathrm{Na}^{+} /$taurocholate cotransporting polypeptide and cholesterol $7 \alpha$-hydroxylase (NTCP) and CYP7A1 were downregulated. The effects of glucose and the FXR antagonist on FXR signaling and lipid metabolism proteins were the opposite of those of bile acid and the FXR agonist.

\section{Discussion}

Liver regeneration is crucial for patients who undergo partial hepatectomy or liver transplantation. It is therefore important to investigate methods of improving the capacity of the liver to regenerate in response to damage. A number of factors are involved in liver regeneration, including a variety of cytokines and growth factors. Previous studies have shown that bile acid and FXR are required for early liver regeneration $(17,18)$. In vitro studies have demonstrated that physiological concentrations of bile acid promote hepatocyte proliferation (19). However, excess bile acid cause degeneration and necrosis of liver cells $(19,20)$. In one study, fatalities occurred in mice that had been fed with $1 \%$ bile acid and subjected to $70 \%$ hepatectomy, suggesting that bile acid not only failed to promote liver regeneration, but was likely to be cytotoxic, perhaps as a result of the higher dose to that employed in the present study (21). Furthermore, the mRNA expression of FXR in 2/3 hepatectomy rats was significantly increased, and the hepatic regeneration rate of FXR-knockout mice subjected to $70 \%$ hepatectomy, was shown to be inhibited (11). In addition, FXR alleviated age-related proliferation defects by activating Fork head Box mlb transcription in regenerating mouse livers (22), and was also shown to regulate liver repair following $\mathrm{CCl}_{4}$-induced toxic injury (23). Borude et al (24) demonstrated that hepatocyte-specific deletion of FXR delayed, but did not completely inhibit, liver regeneration following partial hepatectomy, by delaying cyclin D1 activation. Another study suggested that hepatic-FXR and intestinal-FXR participate in the promotion of liver regeneration and repair in mice (25). All studies have shown that bile acid and FXR are important in the process of liver regeneration. Our study showed that $0.2 \%$ bile acid significantly increased the liver growth of rats that 
had undergone hepatectomy and that this result was reversed in the glucose group, as indicated by the level of expression of PCNA. PCNA is a marker for DNA synthesis that acts as a scaffold for DNA-related enzymes by encircling dsDNA and is commonly used as an indicator of cell proliferation $(26,27)$. In addition, $0.2 \%$ bile acid increased the serum and liver bile acid levels, demonstrating that bile acid is associated with improved liver regeneration following major hepatectomy. The protein levels of FXR and Caveolin-1 were found to be elevated in the bile acid group and reduced in the glucose group. Caveolin-1 is a structural protein of caveolae, which is a subtype of cholesterol-enriched lipid microdomains that are usually observed as vesicles pinching off from the plasma membrane (28). Caveolin-1 has previously been reported to regulate lipid metabolism, apoptosis and endocytosis in cells $(29,30)$. In the present study, administration of bile acid upregulated the expression of caveolin-1 and the hepatic regeneration rate, which was consistent with previous research.

This study provided evidence that bile acid and FXR are involved in liver regeneration. TG, TC, HDL, LDL and FFA levels in liver cells were then compared in vitro. Primary cultured hepatocytes from rats were treated with bile acid, glucose, FXR agonist or FXR antagonist, and changes in lipid metabolism factors were measured. The results indicated that bile acid and FXR agonists reduced the levels of TG, LDL and FFA, and increased the levels of TC and HDL. In the subsequent study, examining the expression of FXR signaling-related proteins in hepatocytes, the mechanisms underlying the effects of bile acid and FXR on lipid metabolism and liver regeneration were investigated. Activated FXR stimulated expression of SHP, which integrated with the liver receptor homolog and inhibited transcription of CYP7A1 $(31,32)$. Bile acid is known to be a regulator of cysteine sulfinic acid decarboxylase via mechanisms shared in part with CYP7A1, and may affect cholesterol via CYP7A1 through the downregulation of the hepatic FXR/SHP pathway $(33,34)$. Bile acid levels were shown to be increased with an increased expression of BSEP and the expression of NTCP and ASBT are also known to be involved in the regulation of bile acid metabolism $(35,36,37)$. In the present study, the expression of BSEP, Caveolin-1 and SHP significantly increased, while that of ASBT, NTCP and CYP7A1 decreased, in accordance with previous studies.

In conclusion, the current study demonstrated that bile acid and FXR are involved in the regulation of liver regeneration, and may affect the lipid metabolism and glycometabolism of the liver. In view of these properties, it is possible that bile acid regulates energy metabolism through FXR signaling pathways and that physiological concentrations of bile acid promote liver regeneration.

\section{Acknowledgements}

This study was supported by a grant from the National Natural Science Foundation of China (grant no. 81141047).

\section{References}

1. Fausto N, Campbell JS and Riehle KJ: Liver regeneration. Hepatology 43 (2 Suppl 1): S45-S53, 2006.
2. Mohammed FF and Khokha R: Thinking outside the cell: proteases regulate hepatocyte division. Trends Cell Biol 15: $555-563,2005$.

3. Gerbino A, Ranieri M, Lupo S, et al: $\mathrm{Ca}^{+}$-dependent $\mathrm{K}^{+}$efflux regulates deoxycholate-induced apoptosis of BHK-21 and Caco-2 cells. Gastroenterology 137: 955-964, e951-e952, 2009.

4. Drudi Metalli V, Mancino MG, Mancino A, et al: Bile salts regulate proliferation and apoptosis of liver cells by modulating the IGF1 system. Dig Liver Dis 39: 654-662, 2007.

5. Staudinger JL, Woody S, Sun M and Cui W: Nuclear-receptor-mediated regulation of drug- and bile-acid-transporter proteins in gut and liver. Drug Metab Rev 45: 48-59, 2013.

6. Carlberg C and Seuter S: Dynamics of nuclear receptor target gene regulation. Chromosoma 119: 479-484, 2010.

7. Forman BM, Goode E, Chen J, et al: Identification of a nuclear receptor that is activated by farnesol metabolites. Cell 81: 687-693, 1995.

8. Wang YD, Chen WD, Moore DD and Huang W: FXR: a metabolic regulator and cell protector. Cell Res 18: 1087-1095, 2008.

9. Gadaleta RM, van Mil SW, Oldenburg B, Siersema PD, Klomp LW and van Erpecum KJ: Bile acids and their nuclear receptor FXR: Relevance for hepatobiliary and gastrointestinal disease. Biochim Biophys Acta 1801: 683-692, 2010

10. Peterson DF, Coote JH, Gilbey MP and Futuro-Neto HA: Differential pattern of sympathetic outflow during upper airway stimulation with smoke. Am J Physiol 245: R433-R437, 1983.

11. Xing X, Burgermeister E, Geisler F, et al: Hematopoietically expressed homeobox is a target gene of farnesoid $\mathrm{X}$ receptor in chenodeoxycholic acid-induced liver hypertrophy. Hepatology 49: 979-988, 2009.

12. Lefebvre P, Cariou B, Lien F, Kuipers F and Staels B: Role of bile acids and bile acid receptors in metabolic regulation. Physiol Rev 89: 147-191, 2009.

13. El-Shenawy NS: Effects of insecticides fenitrothion, endosulfan and abamectin on antioxidant parameters of isolated rat hepatocytes. Toxicol In Vitro 24: 1148-1157, 2010.

14. Matsubara T, Li F and Gonzalez FJ: FXR signaling in the enterohepatic system. Mol Cell Endocrinol 368: 17-29, 2013.

15. Fernández MA, Albor C, Ingelmo-Torres M, et al: Caveolin-1 is essential for liver regeneration. Science 313: 1628-1632, 2006.

16. Hoekstra LT, van Lienden KP, Schaap FG, Chamuleau RA, Bennink RJ and van Gulik TM: Can plasma bile salt, triglycerides, and apoA-V levels predict liver regeneration? World J Surg 36: 2901-2908, 2012.

17. Csanaky IL, Aleksunes LM, Tanaka Y and Klaassen CD: Role of hepatic transporters in prevention of bile acid toxicity after partial hepatectomy in mice. Am J Physiol Gastrointest Liver Physiol 297: G419-G433, 2009.

18. Otao R, Beppu T, Isiko T, et al: External biliary drainage and liver regeneration after major hepatectomy. Br J Surg 99: 1569-1574, 2012

19. Perez MJ and Briz O: Bile-acid-induced cell injury and protection. World J Gastroenterol 15: 1677-1689, 2009.

20. Kren BT, Rodrigues CM, Setchell KD and Steer CJ: Modulation of steady-state messenger RNA levels in the regenerating rat liver with bile acid feeding. Liver Transpl 7: 321-334, 2001.

21. Zhang L, Huang X, Meng Z, et al: Significance and mechanism of CYP7a1 gene regulation during the acute phase of liver regeneration. Mol Endocrinol 23: 137-145, 2009.

22. Chen WD, Wang YD, Zhang L, et al: Farnesoid X receptor alleviates age-related proliferation defects in regenerating mouse livers by activating forkhead box m1b transcription. Hepatology 51: 953-962, 2010.

23. Meng Z, Wang Y, Wang L, et al: FXR regulates liver repair after CCl4-induced toxic injury. Mol Endocrinol 24: 886-897, 2010.

24. Borude P, Edwards G, Walesky C, et al: Hepatocyte-specific deletion of farnesoid $\mathrm{X}$ receptor delays but does not inhibit liver regeneration after partial hepatectomy in mice. Hepatology 56: 2344-2352, 2012.

25. Zhang L, Wang YD, Chen WD, et al: Promotion of liver regeneration/repair by farnesoid $\mathrm{X}$ receptor in both liver and intestine in mice. Hepatology 56: 2336-2343, 2012.

26. Dionne I, Brown NJ, Woodgate R and Bell SD: On the mechanism of loading the PCNA sliding clamp by RFC. Mol Microbiol 68: 216-222, 2008

27. Hlinkova V, Xing G, Bauer J, et al: Structures of monomeric, dimeric and trimeric PCNA: PCNA-ring assembly and opening. Acta Crystallogr D Biol Crystallogr 64: 941-949, 2008. 
28. Woudenberg J, Rembacz KP, van den Heuvel FA, et al: Caveolin-1 is enriched in the peroxisomal membrane of rat hepatocytes. Hepatology 51: 1744-1753, 2010.

29. Fernández-Rojo MA, Restall C, Ferguson C, et al: Caveolin-1 orchestrates the balance between glucose and lipid-dependent energy metabolism: implications for liver regeneration. Hepatology 55: 1574-1584, 2012.

30. Meyer C, Liu Y, Kaul A, Peipe I and Dooley S: Caveolin-1 abrogates TGF-beta mediated hepatocyte apoptosis. Cell Death Dis 4: e466, 2013.

31. Li G, Thomas AM, Hart SN, Zhong X, Wu D and Guo GL: Farnesoid $\mathrm{X}$ receptor activation mediates head-to-tail chromatin looping in the NrOb2 gene encoding small heterodimer partner. Mol Endocrinol 24: 1404-1412, 2010.

32. Anakk S, Watanabe M, Ochsner SA, McKenna NJ, Finegold MJ and Moore DD: Combined deletion of FXR and SHP in mice induces Cyp17a1 and results in juvenile onset cholestasis. J Clin Invest 121: 86-95, 2011.
33. Kerr TA, Matsumoto Y, Matsumoto H, et al: Cysteine sulfinic acid decarboxylase regulation: A role for farnesoid $\mathrm{X}$ receptor and small heterodimer partner in murine hepatic taurine metabolism. Hepatol Res 44: E218-E228, 2013.

34. Matsui S, Yamane T, Takita T, Oishi Y and Kobayashi-Hattori K: The hypocholesterolemic activity of Momordica charantia fruit is mediated by the altered cholesterol- and bile acid-regulating gene expression in rat liver. Nutr Res 33: 580-585, 2013.

35. Zhu QN, Xie HM, Zhang D, Liu J and Lu YF: Hepatic bile acids and bile acid-related gene expression in pregnant and lactating rats. Peer J 1: e143, 2013.

36. Miura T, Kimura N, Yamada T, et al: Sustained repression and translocation of Ntcp and expression of Mrp4 for cholestasis after rat 90\% partial hepatectomy. J Hepatol 55: 407-414, 2011.

37. Hoang MH, Houng SJ, Jun HJ, et al: Barley intake induces bile acid excretion by reduced expression of intestinal ASBT and NPC1L1 in C57BL/6 J mice. J Agric Food Chem 59: 6798-6805, 2011. 